\title{
Amplitudes, recursion relations and unitarity in the Abelian Higgs model
}

\section{Ronald Kleiss and Oscar Boher Luna}

Institute for Mathematics, Astrophysics and Particle Physics, Radboud University Nijmegen, Heyendaalseweg 135, Nijmegen, the Netherlands

E-mail: R.Kleiss@science.ru.nl, oscar_bl92@hotmail.com

ABSTRACT: The Abelian Higgs model forms an essential part of the electroweak standard model: it is the sector containing only $\mathrm{Z}^{0}$ and Higgs bosons. We present a diagrambased proof of the tree-level unitarity of this model inside the unitary gauge, where only physical degrees of freedom occur. We derive combinatorial recursion relations for off-shell amplitudes in the massless approximation, which allows us to prove the cancellation of the first two orders in energy of unitarity-violating high-energy behaviour for any tree-level amplitude in this model. We describe a deformation of the amplitudes by extending the physical phase space to at least 7 spacetime dimensions, which leads to on-shell recursion relations à la BCFW. These lead to a simple proof that all on-shell tree amplitudes obey partial-wave unitarity.

KeYwords: Scattering Amplitudes, Higgs Physics

ArXiv EPrint: 1705.04859 


\section{Contents}

1 Introduction 1

2 The $\mathrm{ZH}$ sector in the unitary gauge 3

3 Recursion relations for off-shell amplitudes 4

4 Mass effects and transversality $\quad 7$

5 On-shell recursion relations $\quad 10$

6 Conclusions 13

\section{Introduction}

The Minimal Standard Model includes a few sectors that are consistent theories in their own right. The best-known of these are QED and QCD, but in addition we have the $\mathrm{ZH}$ sector: it contains only the $\mathrm{Z}$ and the $\mathrm{H}$ bosons, with their interactions. This is, in fact, nothing but the Abelian Higgs model [1], the simplest example of a spontaneously broken gauge symmetry in quantum field theory. The internal consistency of such models is of course uncontroversial [2]. In particular they respect unitarity, with which we mean the behaviour of on-shell scattering amplitudes with energy when all masses and scattering angles are kept constant: it implies unitarity in each partial wave separately. This is not trivial, especially because of the longitudinal degrees of freedom present in massive spin-1 particles. The bestknown proof of perturbative unitarity of the Abelian Higgs model is presented [3]. In that paper, the authors point out that unphysical fields must be involved in the case of a general gauge, and the main issue is to get rid of these fields, and of the gauge dependence, in the finally resulting S-matrix elements. Other proofs, like that of the equivalence theorem [4] also typically rely on the Feynman-'t Hooft gauge. There is, however, another way to view the Abelian Higgs model. Rather than starting with the unbroken theory, which is a gauge theory, we may as well simply regard the broken Lagrangian 'as given', that is a theory containing two massive particles, with spins 0 and 1 , without worrying where it came from. Massive spin-1 theories do not suffer from the necessity of fixing a gauge, since there is no gauge symmetry. It ought therefore to be possible to prove unitarity of the amplitudes directly using only the physical fields, with the Proca propagator, ${ }^{1}$ at least at the tree level. In higher loops (which we do not consider here), the effect of the Faddeev-Popov ghosts can be implemented by introducing counterterms proportional to the space-time volume,

\footnotetext{
${ }^{1}$ In gauge theory language, the unitary gauge.
} 
as described e.g. in [5]. ${ }^{2}$ This is the approach we adopt here: we shall use only the physical $\mathrm{Z}$ and $\mathrm{H}$ fields, and the unitary-gauge propagator for the $\mathrm{Z}$.

The study of multi-leg amplitudes is a flourishing field. Excellent didactic reviews are for instance $[6,7]$. These mainly discuss theories with a high degree of symmetry (with $N=4$ super-Yang-Mills as an extreme example), whereas we are dealing with a theory with very little symmetry, and with explicitly massive particles. In addition, the approach of choice is to express all fields in terms of massless (Weyl) spinors and employ the arsenal of techniques available for such formulations. In the spirit of the previous paragraph, we hold that it ought to be possible to restrict ourselves to (scalar and) vector fields only: no spinors will intrude in our derivations.

Partial-wave unitarity requires cross sections at some energy scale $E$ to decrease as $E^{-2}$ when $E$ becomes large, and all angles are kept fixed [8,9]. Since for an $n$-point amplitude $\mathcal{M}_{n}$, relevant to $2 \rightarrow(n-2)$ processes, the concomitant phase space has dimensionality ${ }^{3}$ $E^{2 n-8}$, acceptable unitarity (high-energy) behaviour implies

$$
\mathcal{M}_{n} \sim E^{4-n}
$$

at high energies. As we shall show, power counting gives us a behaviour up to $E^{+2}$ for amplitudes in the ZH sector, so cancellations over many orders of magnitude (powers of $E$ over some mass) must occur for large- $n$ amplitudes. At the tree level, an amplitude like that for $\mathrm{ZZ} \rightarrow 4 \mathrm{Z}+6 \mathrm{H}$ is based on $649,271,700$ diagrams and calls for a cancellation over 10 orders of magnitude: clearly we must be as systematic as possible.

Our strategy in this paper will be as follows. We shall first establish effective Feynman rules that describe off-shell amplitudes at the $E^{2}$ level, that is, the most dangerous behaviour with energy. The Schwinger-Dyson equations (SDe) of the model provide recursion relations between these amplitudes which have surprisingly simple solutions. The vanishing of the $E^{2}$ terms is then immediately obvious. The $E^{0}$ terms can be obtained from these off-shell amplitudes by including the effects of nonzero masses in a perturbative approach, and we shall show that in first order these vanish as well, provided that the Higgs selfinteractions are correctly chosen. We then turn to on-shell recursion relations, that deal with the splitting-up of amplitudes into products of lesser on-shell amplitudes connected by off-shell propagators. The less-than- $E^{0}$ behaviour of these amplitudes then allows us to prove the unitarity of all tree-level amplitudes. For this it will turn out to be necessary to deform the momenta (and polarisations) of the particles by extending the four-dimensional phase space of actual physics to a higher-dimensional one; fortunately, since no spinors are involved the technicalities of this deformation are fairly straightforward.

\footnotetext{
${ }^{2}$ These counterterms arise from the infinite-momentum limit of 1PI diagrams where a closed Z loop couples to an arbitrary number of $\mathrm{H}$ legs, thus giving rise to a non-polynomial Higgs counterterm Lagrangian.

${ }^{3}$ Each of the $n-2$ final-state momenta contributes $E^{2}$, and the delta function imposing four-momentum conservation scales as $E^{-4}$.
} 


\section{The $\mathrm{ZH}$ sector in the unitary gauge}

The propagator of the $\mathrm{Z}$ and $\mathrm{H}$ bosons are given by, respectively,

$$
\begin{aligned}
\mu \ldots \ldots \ldots v & =\frac{i}{p^{2}-m^{2}}\left(-g^{\mu \nu}+\frac{1}{m^{2}} p^{\mu} p^{\nu}\right) \\
& =\frac{-i}{p^{2}-m^{2}} T^{\mu \nu}(p)+\frac{i}{m^{2}} L^{\mu \nu}(p), \\
\frac{\mathrm{p}}{p^{2}-M^{2}} . & =\frac{i}{}
\end{aligned}
$$

Here

$$
T^{\mu \nu}(p)=g^{\mu \nu}-p^{\mu} p^{\nu} / p^{2}, \quad L^{\mu \nu}(p)=p^{\mu} p^{\nu} / p^{2}
$$

are the two purely transverse and longitudinal projection tensors, with $T^{2}=T, L^{2}=L$ and $T L=0$. The mass of the $\mathrm{Z}$ and of the Higgs are denoted by $m$ and $M$, respectively. By either reading them off from the electroweak Lagrangian, ${ }^{4}$ or by insisting on correct high-energy behaviour of the amplitudes ${ }^{5} \mathcal{M}(2 \mathrm{Z}, 2 \mathrm{H}), \mathcal{M}(2 \mathrm{Z}, 3 \mathrm{H})$ and $\mathcal{M}(4 \mathrm{Z}, 1 \mathrm{H})[11],{ }^{6}$ we establish the Feynman rules for the vertices:
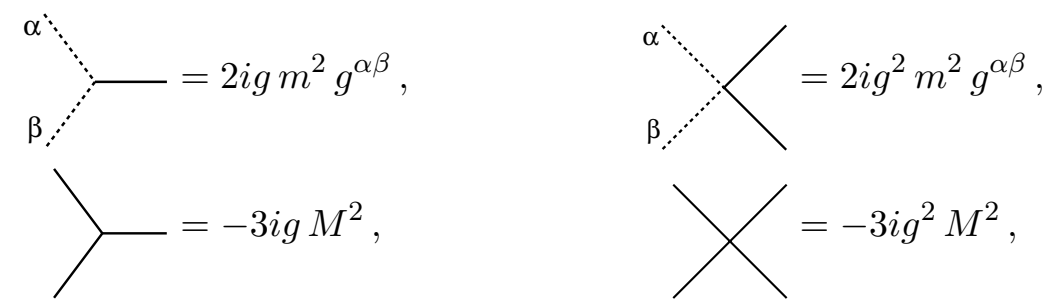

where

$$
g^{2}=G_{F} \sqrt{2},
$$

and $G_{F}$ is the Fermi coupling constant. For an external $\mathrm{Z}$ the longitudinal polarisation vector can be constructed as

$$
\epsilon_{L}^{\mu}=\frac{1}{m}\left(q^{\mu}-\frac{m^{2}}{(q \cdot t)} t^{\mu}\right)
$$

where $t$ is a lightlike vector which we shall choose to be the same for all Z's in the amplitude. For an arbitrary Minkowski vector $r$, the projection

$$
r^{\mu} \rightarrow r^{\mu}+\frac{m^{2}(r \cdot t)-(r \cdot q)(q \cdot t)}{(q \cdot t)^{2}} t^{\mu}-\frac{(r \cdot t)}{(q \cdot t)} q^{\mu}
$$

gives an vector orthogonal to both $q$ and $t$, that can then be normalized to a transverse polarisation vector $\epsilon_{T}$. This is especially useful for Monte Carlo investigation of the amplitudes.

\footnotetext{
${ }^{4} \mathrm{~A}$ most complete set of Feynman rules is given in e.g. ref. [10].

${ }^{5} \mathrm{By} \mathcal{M}(p \mathrm{Z}, q \mathrm{H})$ we denote the tree-level amplitude with $p$ external $\mathrm{Z}$ bosons and $q$ external $\mathrm{H}$ bosons.

${ }^{6}$ This approach follows that of refs. $[12,13]$.
} 


\section{Recursion relations for off-shell amplitudes}

In order to maximize the power-counting behaviour with $E$ for any amplitude in the $\mathrm{ZH}$ sector, we must choose all external Z's to have longitudinal polarisation; we must use the $L$ part of the $\mathrm{Z}$ propagators; and we must reduce the number of $\mathrm{H}$ propagators to a minimum. This implies that diagrams with Higgs self-interactions are always of lower order in $E$. It is then easily checked that the highest possible $E$ dependence in any tree amplitude is $E^{2}$. Since all diagrams in an $n$-point tree amplitude have the same power $g^{n-2}$ we may put $g=1$ for simplicity. If we adopt the convention that all external on-shell momenta are counted outgoing, we may replace the original Feynman rules by the following ones:

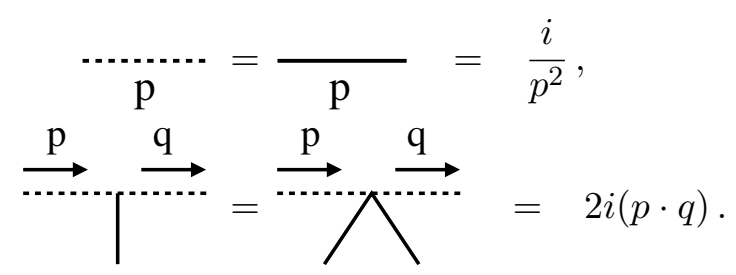

All external (on-shell) lines carry a trivial factor 1 in this formulation; also implied is an overall factor $(-)^{n / 2}$ in an on-shell amplitude with $n$ external $\mathrm{Z}$ bosons. The ZH model in this limit is a theory with two massless scalars and a derivative coupling. We shall compute the off-shell amplitude for a $Z$ or a $\mathrm{H}$ going to $n$ Z's and $k$ H's:
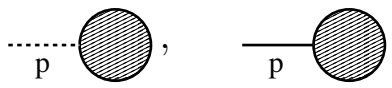

which we denote by $Z_{n, k}$ and $H_{n, k}$, respectively. The amplitude includes the off-shell propagator, and the momentum $p$ is counted going into the diagrams. The outgoing Higgs momenta are denoted $h_{i}, i=1, \ldots, k$, and $h=h_{1}+\cdots+h_{k}$; the outgoing $\mathrm{Z}$ momenta are denoted by $q_{i}, i=1, \ldots, n$, and $q=q_{1}+\cdots+q_{n}$.

By explicit calculation for several modest values of $n$ and $k$ we can arrive at the following conjecture, which we shall prove:

$$
\begin{aligned}
Z_{n, k} & =\left\{\begin{array}{lr}
(-)^{k}(n-1+k) !, & n \text { odd } \\
0, & n \text { even }
\end{array},\right. \\
H_{n, k} & =\left\{\begin{array}{lr}
1, & n=0, k=1 \\
(-)^{k+1}(n-2+k) ! \gamma_{n}, & n \geq 2 \text { even } \\
0, & n \text { odd }
\end{array},\right. \\
\gamma_{n} & =\left\{\begin{array}{lr}
1, & n=2 \\
(n-1) ! !(n-3) ! ! /(n-2) !, & n \geq 4 \text { even }
\end{array}\right.
\end{aligned} .
$$

These values can conveniently be gathered into two generating functions:

$$
\begin{aligned}
\zeta=\zeta(x, y) & =\sum_{k, n \geq 0} \frac{x^{n} y^{k}}{n ! k !} Z_{n, k}=\frac{1}{2} \log \left(\frac{1+y+x}{1+y-x}\right), \\
\chi=\chi(x, y) & =\sum_{k, n \geq 0} \frac{x^{n} y^{k}}{n ! k !} H_{n, k}=-1+\sqrt{(1+y)^{2}-x^{2}} .
\end{aligned}
$$


The SDe for the simplified model giving the $E^{2}$ terms read

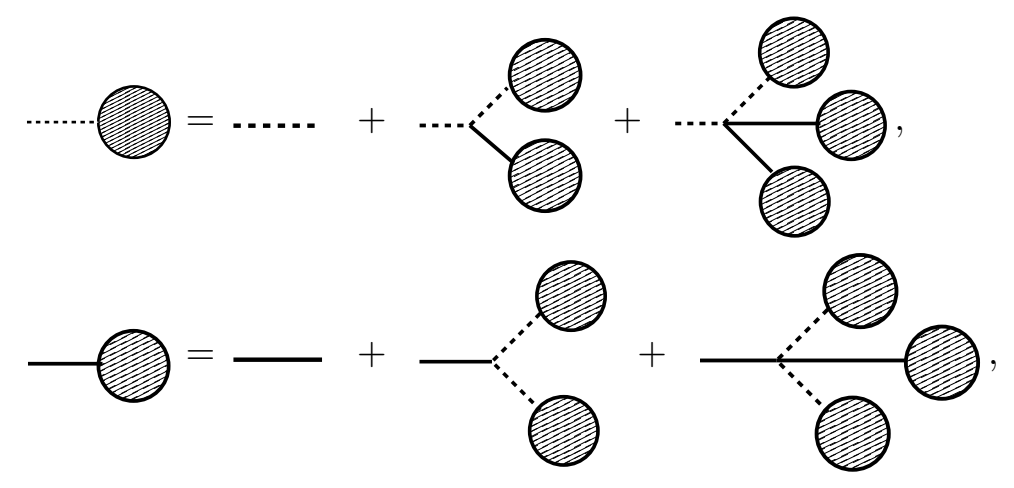

The best calculational strategy is to realise that, in any of our off-shell amplitudes, the coefficient of $\left(q_{1} \cdot q_{2}\right)$ must be equal to that of $q^{2} / 2$ in the final expression owing to the symmetry between the Z's. Similarly the coefficient of $\left(h_{1} \cdot h_{2}\right)$ gives that of $h^{2} / 2$, and the coefficient of $\left(q_{1} \cdot h_{1}\right)$ gives that of $(q \cdot h)$. We therefore only have to keep track of a few momentum products to be able to reproduce the whole off-shell amplitude $H_{n, k}$. Similarly, the coefficient of $\left(p \cdot q_{1}\right)$ is that of $(p \cdot q)$, and the coefficient of $\left(p \cdot h_{1}\right)$ is that of $(p \cdot h)$ in the $Z_{n, k}$. We can write the off-shell amplitudes as ${ }^{7}$

$$
\begin{aligned}
& Z_{n, k}=\theta((n, k)=(1,0))-\frac{2}{p^{2}}\left((p \cdot q) A_{n, k}^{(1)}+(p \cdot h) A_{n, k}^{(2)}\right) \\
& H_{n, k}=\theta((n, k)=(0,1))-\frac{2}{p^{2}}\left(\frac{1}{2} q^{2} A_{n, k}^{(3)}+\frac{1}{2} h^{2} A_{n, k}^{(4)}+(q \cdot h) A_{n, k}^{(5)}\right) .
\end{aligned}
$$

The several $A$ 's are given by

$$
\begin{aligned}
A_{n, k}^{(1)}= & \sum_{m, \ell}\left(\begin{array}{c}
n-1 \\
m-1
\end{array}\right)\left(\begin{array}{c}
k \\
\ell
\end{array}\right) Z_{m, \ell} H_{n-m, k-\ell} \\
& +\frac{1}{2} \sum_{m, t, \ell, r}\left(\begin{array}{c}
n-1 \\
m-1, t
\end{array}\right)\left(\begin{array}{c}
k \\
\ell, r
\end{array}\right) Z_{m, \ell} H_{t, r} H_{n-m-t, k-\ell-r}, \\
A_{n, k}^{(2)}= & \sum_{m, \ell}\left(\begin{array}{c}
n \\
m
\end{array}\right)\left(\begin{array}{c}
k-1 \\
\ell-1
\end{array}\right) Z_{m, \ell} H_{n-m, k-\ell} \\
& +\frac{1}{2} \sum_{m, \ell, t, r}\left(\begin{array}{c}
n \\
m, t
\end{array}\right)\left(\begin{array}{c}
k-1 \\
\ell-1, r
\end{array}\right) Z_{m, \ell} H_{t, r} H_{n-m-t, k-\ell-r}, \\
A_{n, k}^{(3)}= & \sum_{m, \ell}\left(\begin{array}{c}
n-2 \\
m-1
\end{array}\right)\left(\begin{array}{c}
k \\
\ell
\end{array}\right) Z_{m, \ell} Z_{n-m, k-\ell} \\
& +\sum_{m, \ell, t, r}\left(\begin{array}{c}
n-2 \\
m-1, t-1
\end{array}\right)\left(\begin{array}{c}
k \\
\ell, r
\end{array}\right) Z_{m, \ell} Z_{t, r} H_{n-m-t, k-\ell-r},
\end{aligned}
$$

\footnotetext{
${ }^{7}$ The logical step function $\theta(\mathcal{P})$ is 1 if $\mathcal{P}$ is true, 0 if $\mathcal{P}$ is false.
} 


$$
\begin{aligned}
A_{n, k}^{(4)}= & \sum_{m, \ell}\left(\begin{array}{c}
n \\
m
\end{array}\right)\left(\begin{array}{c}
k-2 \\
\ell-1
\end{array}\right) Z_{m, \ell} Z_{n-m, k-\ell} \\
& +\sum_{m, \ell, t, r}\left(\begin{array}{c}
n \\
m, t
\end{array}\right)\left(\begin{array}{c}
k-2 \\
\ell-1, r-1
\end{array}\right) Z_{m, \ell} Z_{t, r} H_{n-m-t, k-\ell-r}, \\
A_{n, k}^{(5)}= & \sum_{m, \ell}\left(\begin{array}{c}
n-1 \\
m-1
\end{array}\right)\left(\begin{array}{c}
k-1 \\
\ell-1
\end{array}\right) Z_{m, \ell} Z_{n-m, k-\ell} \\
& +\sum_{m, \ell, t, r}\left(\begin{array}{c}
n-1 \\
m-1, t
\end{array}\right)\left(\begin{array}{c}
k-1 \\
\ell, r-1
\end{array}\right) Z_{m, \ell} Z_{t, r} H_{n-m-t, k-\ell-r} .
\end{aligned}
$$

The summations run from 0 to infinity. Throughout this paper we use the convention

$$
\left(\begin{array}{l}
a \\
b
\end{array}\right)=0 \text { for } b<0 \text { or } b>a
$$

since $(-n)$ ! diverges for natural numbers $n>0$, and the definitions

$$
\left(\begin{array}{c}
a \\
b, c
\end{array}\right)=\left(\begin{array}{l}
a \\
b
\end{array}\right)\left(\begin{array}{c}
a-b \\
c
\end{array}\right), \quad\left(\begin{array}{c}
a \\
b, c, d
\end{array}\right)=\left(\begin{array}{l}
a \\
b
\end{array}\right)\left(\begin{array}{c}
a-b \\
c
\end{array}\right)\left(\begin{array}{c}
a-b-c \\
d
\end{array}\right) .
$$

To illustrate how the combinatorial factors come about, let us look at the first term in the recursive expression for $\mathcal{A}^{(1)}$ in eq. (3.6). The coefficient of $(p \cdot q)$ is, as we have argued, that of $\left(p \cdot q_{1}\right)$. Now the vector $q_{1}$ can only come from the Z. In the object $Z_{m, \ell}$ we therefore single out the vector $q_{1}$, and then there are $m-1$ other external $\mathrm{Z}$ momenta left, to be chosen from $n-1$ available ones; this gives the first binomial. The second binomial comes from the number of ways to choose $\ell \mathrm{H}$ momenta out of $k$. We stress that we do not assume the momenta $q_{j}$ to be all equal, as is done in studies of threshold amplitudes; $;^{8,9}$ rather, we use the fact that amplitudes must be symmetric in the $q$ 's as well as in the $h$ 's.

By computer algebra the relations (3.5) can be checked for different values of $(n, k)$; but it is more profitable to inspect the generating functions of the $A$ 's,

$$
\mathcal{A}^{(j)}(x, y)=\sum_{n, k \geq 0} \frac{x^{n} y^{k}}{n ! k !} A_{n, k}^{(j)}, \quad j=1, \ldots, 5 .
$$

The functions $\mathcal{A}^{(1,2)}$ must be odd in $x$, the functions $\mathcal{A}^{(3,4,5)}$ even. The SDe take the following forms:

$$
\begin{aligned}
& \zeta=x-2 \mathcal{A}^{(1)} \frac{(p \cdot q)}{p^{2}}-2 \mathcal{A}^{(2)} \frac{(p \cdot h)}{p^{2}}, \\
& \chi=y-\mathcal{A}^{(3)} \frac{q^{2}}{p^{2}}-\mathcal{A}^{(4)} \frac{h^{2}}{p^{2}}-2 \mathcal{A}^{(5)} \frac{(q \cdot h)}{p^{2}} .
\end{aligned}
$$

Since we already have our conjecture on the form of $\zeta$ and $\chi$ we only need to establish the consistency of these equations rather than provide an all-out proof, because given the

\footnotetext{
${ }^{8}$ For example in refs. [14-16].

${ }^{9}$ For a recent application see for instance ref. [17].
} 
correct starting points the recursion relations lead to unique answers. We can easily derive the following differential relations:

$$
\begin{aligned}
-2 \frac{\partial}{\partial x} \mathcal{A}^{(1)} & =-2\left(\frac{\partial}{\partial x} \zeta\right)\left(\chi+\frac{1}{2} \chi^{2}\right)=\frac{\partial}{\partial x} \zeta-1-y, \\
-2 \frac{\partial}{\partial y} \mathcal{A}^{(2)} & =-2\left(\frac{\partial}{\partial y} \zeta\right)\left(\chi+\frac{1}{2} \chi^{2}\right)=\frac{\partial}{\partial y} \zeta+x, \\
-\frac{\partial^{2}}{(\partial x)^{2}} \mathcal{A}^{(3)} & =-\left(\frac{\partial}{\partial x} \zeta\right)^{2}(1+\chi)=\frac{\partial^{2}}{(\partial x)^{2}} \chi, \\
-\frac{\partial^{2}}{(\partial y)^{2}} \mathcal{A}^{(4)} & =-\left(\frac{\partial}{\partial y} \zeta\right)^{2}(1+\chi)=\frac{\partial^{2}}{(\partial y)^{2}} \chi, \\
-2 \frac{\partial}{\partial x} \frac{\partial}{\partial y} \mathcal{A}^{(5)} & =-2\left(\frac{\partial}{\partial x} \zeta\right)\left(\frac{\partial}{\partial y} \zeta\right)(1+\chi)=2 \frac{\partial}{\partial x} \frac{\partial}{\partial y} \chi .
\end{aligned}
$$

Using the even/odd properties of the $\mathcal{A}$ 's we arrive at

$$
\begin{aligned}
-2 \mathcal{A}^{(1)} & =\zeta-x-x y, \\
-2 \mathcal{A}^{(2)} & =\zeta+x y+f_{1}(x), \\
-\mathcal{A}^{(3)} & =\chi+f_{2}(y), \\
-\mathcal{A}^{(4)} & =\chi+y f_{3}(x)+f_{4}(x), \\
-2 \mathcal{A}^{(5)} & =2 \chi+f_{5}(x)+f_{6}(y) .
\end{aligned}
$$

Here the functions $f_{j}(j=1, \ldots, 6)$ are undetermined. Note, however, that the terms with $f_{1,3,4,5}$ correspond to either $h^{\mu}=0$ or $h^{2}=0$, while those with $f_{2,6}$ correspond to $q^{\mu}=0$. These terms therefore effectively vanish. A sole exception is the possibility $f_{3}(x)=$ constant. The starting value $H_{0,1}=1$ tells us to take $f_{3}(x)=-1$, so eq. (3.11) is satisfied. The right-hand side of eq. (3.10) reads

$$
\zeta+x\left(1-\frac{(p \cdot q)}{p^{2}}\right)+x y\left(\frac{(p \cdot q)}{p^{2}}-\frac{(p \cdot h)}{p^{2}}\right) .
$$

For $(n, k)=(1,0)$ we have $p \cdot q=p^{2}$, and for $(n, k)=(1,1)$ we have $p \cdot q=p \cdot h=p^{2} / 2$, so that the extra terms also effectively vanish. This establishes the correctness of the conjecture (3.2). Since the off-shell amplitudes do not depend on $p^{2}$ we have now proven that in all on-shell amplitudes the $E^{2}$ terms vanish.

\section{Mass effects and transversality}

So far we have taken the $\mathrm{Z}$ and $\mathrm{H}$ bosons to be massless, which was appropriate for examining the $E^{2}$ terms. In the next order, $E^{0}$, we have to consider the effects of nonzero masses. These come from different sources. For the $\mathrm{Z}$ mass $m$ we have the effect of $q_{j}^{2}=m^{2}$ for external Z's; the correction term with $t^{\mu}$ for longitudinally polarised bosons in eq. (2.6); and the $T$ term in the $\mathrm{Z}$ propagator. Another possible source of $E^{1}$ or $E^{0}$ terms is the occurrence of one or two transversely polarised $\mathrm{Z}$ bosons. For the Higgs mass $M$ we have 
the effect of $h_{j}^{2}=M^{2}$ for external Higgses; the correction to the Higgs propagator; and the so-far neglected $\mathrm{H}$ self-interactions. To leading order we may inspect all these different effects separately, while keeping to the $E^{2}$ approximation in the rest of the amplitude.

We first deal with the $M^{2}$ terms. The $M^{2}$ corrections in the Higgs propagator can conveniently be included by keeping the propagator massless and including a two-point vertex:

$$
\longrightarrow=-i M^{2} \text {. }
$$

Let us now consider an on-shell amplitude with $n$ Z's and $k$ Higgses. The occurrence of a single 2-,3-, or 4-point vertex gives the $M^{2}$ contribution to the order we are working in, denoted by $M(n, k)$. Keeping track of these vertices and dropping an overall factor $i M^{2}$, we have

$$
\begin{aligned}
M_{n, k}= & \frac{(-1)}{2} \sum_{m, \ell}\left(\begin{array}{c}
n \\
m
\end{array}\right)\left(\begin{array}{c}
k \\
\ell
\end{array}\right) H_{m, \ell} H_{n-m, k-\ell} \\
& +\frac{(-3)}{3 !} \sum_{m, \ell, t, r}\left(\begin{array}{c}
n \\
m, t
\end{array}\right)\left(\begin{array}{c}
k \\
\ell, r
\end{array}\right) H_{m, \ell} H_{t, r} H_{n-m-t, k-\ell-r} \\
& +\frac{(-3)}{4 !} \sum_{m, t, u, \ell, r, s}\left(\begin{array}{c}
n \\
m, t, u
\end{array}\right)\left(\begin{array}{c}
k \\
\ell, r, s
\end{array}\right) H_{m, \ell} H_{t, r} H_{u, s} H_{n-m-t-u, k-\ell-r-s} .
\end{aligned}
$$

The $M^{2}$ contribution from an external Higgs is correctly subsumed in the two-point terms. We immediately find the generating function

$$
\begin{aligned}
\sum_{n, k} \frac{x^{n} y^{k}}{n ! k !} M_{n, k} & =-\frac{1}{2} \chi^{2}-\frac{1}{2} \chi^{3}-\frac{1}{8} \chi^{4} \\
& =-\frac{1}{8} \chi^{2}(2+\chi)^{2}=-\frac{1}{8}\left(2 y+y^{2}-x^{2}\right)^{2} .
\end{aligned}
$$

We find that the $M^{2}$ terms vanish in all on-shell amplitudes except (correctly) the 3 - and 4-point ones, which after all are not required by unitarity to decrease at high energy. We can read off the leading $M^{2}$ terms in these amplitudes:

$$
\begin{array}{lll}
\mathcal{M}(3 H):-3 i M^{2}, & \mathcal{M}(2 Z, 1 H):+i M^{2}, & \\
\mathcal{M}(4 H):-3 i M^{2}, & \mathcal{M}(4 Z):-3 i M^{2}, & \mathcal{M}(2 Z, 2 H):+i M^{2} .
\end{array}
$$

Explicit calculation confirms these results. The last of these is the least trivial one: of the 4 diagrams,
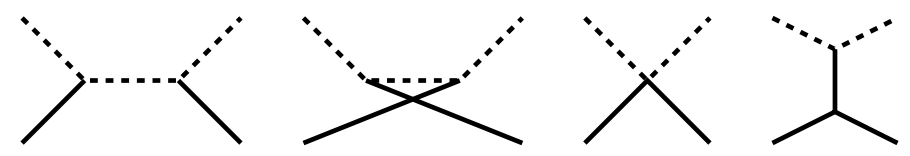

each of the first two ones give $-i M^{2}$, while the fourth diagram contributes $+3 i M^{2}$. We now turn to the $m^{2}$ terms. To this end another recursion relation is needed, namely that 
for off-shell Z's without the propagator factor. These we denote by $K_{n, k}^{\mu}(q)$, where $\mu$ is the uncontracted Lorentz index of the amplitude. We immediately have, at the $E^{2}$ level,

$$
K_{1,0}^{\mu}(q)=q^{\mu}, \quad K_{1,1}^{\mu}=2 i q^{\mu} .
$$

For the other values of $(n, k)$ the recursion relation is diagrammatically also given by the first line of eq. (3.4). Algebraically we therefore have

$$
\begin{aligned}
K_{n, k}^{\mu}(q)= & q^{\mu} \theta(n=1, k=0)+2 i q^{\mu} \theta(n=1, k=1) \\
& +2 i\left(q^{\mu} A_{n, k}^{(1)}+h^{\mu} A_{n, k}^{(2)}\right) \theta(n>1 \text { or } k>1) .
\end{aligned}
$$

In terms of generating functions this reads

$$
\begin{aligned}
\mathcal{K}= & \sum_{n, k} \frac{x^{n} y^{k}}{n ! k !} K_{n, k}^{\mu}(q)=x q^{\mu}+2 i x y q^{\mu} \\
& +2 i q^{\mu}\left(\mathcal{A}^{(1)}-(x, x y)\right)+2 i h^{\mu}\left(\mathcal{A}^{(2)}-(x, x y)\right),
\end{aligned}
$$

where the notation " $-(x, x y)$ " means that the coefficients of both $x$ and $x y$ are to be put to zero. We have

$$
\begin{aligned}
& \mathcal{A}^{(1)}-(x, x y)=\zeta-(x, y), \\
& \mathcal{A}^{(2)}-(x, x y)=\zeta+f_{7}(x)-(x, x y),
\end{aligned}
$$

where, as before, $f_{7}(x)$ is undetermined but refers to cases with $h^{\mu}=0$ anyway. We arrive at

$$
\mathcal{K}=x p^{\mu}+2 i x y q^{\mu}+2 i(\zeta-(x, x y)) p^{\mu} .
$$

With the single exception of the case $n=k=1$, all the amplitudes $K_{n, k}^{\mu}$ are seen to be proportional to the momentum $p^{\mu}$ of the off-shell $Z$ boson. This has two consequences. In the first place, to this order contracting the amplitude $K^{\mu}$ with the $\mathrm{Z}$ polarisation vector $\epsilon_{\mu}$ gives a vanishing result. The only other source of $m^{2}$ terms, the $T$ part of the propagator, is contracted at each end with a $K^{\mu}$ amplitude. This means that it can only survive if it has $K_{1,1}^{\mu}$ at both ends; this implies that the amplitude $\mathcal{M}(2 \mathrm{Z}, 2 \mathrm{H})$ is the only one that has $m^{2} E^{0}$ terms.

From eq. (4.9) we see that potential $E^{1}$ terms in amplitudes with one transversely polarised $\mathrm{Z}$ vanish. If two $\mathrm{Z}$ bosons are transversely polarised the leading terms go as $E^{0}$ by powercounting, and we shall now investigate these by considering amplitudes having two off-shell legs with momenta $q_{a}$ and $q_{b}$, and unresolved Lorentz indices $\alpha$ and $\beta$, respectively, the $n-2$ other Z's being longitudinally polarized. By Lorentz covariance and power counting, such amplitudes must be of the form

$$
i Q g^{\alpha \beta}+i \sum R_{j} \frac{a_{j}{ }^{\alpha} b_{j}{ }^{\beta}}{\Delta_{j}}
$$

where $Q$ and the $R_{j}$ are numbers, $a_{j}$ and $b_{j}$ are (combinations of) momenta, and $\Delta_{j}$ is the denominator of some propagator. 
The only diagrams that contribute to $g^{\alpha \beta}$ in the amplitudes are those where the two off-shell $\mathrm{Z}$ bosons are connected to the same $Z Z H$ or $Z Z H H$ vertex. In terms of the generating functions, we therefore have

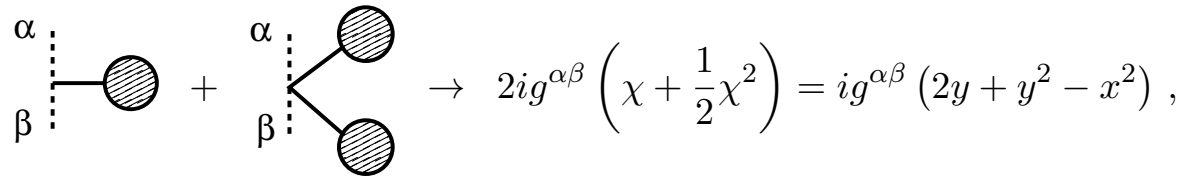

so that $Q=2$ for $\mathcal{M}(2 Z, H)$ and $\mathcal{M}(2 Z, 2 H), Q=-2$ for $\mathcal{M}(4 Z)$, and $Q=0$ for all other amplitudes.

The $R_{j}$ correspond to the residues of the poles of the propagators $1 / \Delta_{j}$. Since neither the $Z_{n, k}$ nor the $H_{n, k}$ have poles, $\Delta$ 's that contain either none or both the off-shell Z's do not contribute to the $E^{0}$ terms. Let us consider the doubly off-shell amplitude $\mathcal{M}\left(q_{a}^{\alpha}, q_{b} \beta, q_{1}, q_{2}, \ldots, q_{n-2}, h_{1}, h_{2}, \ldots, h_{k}\right)$ which has $n \mathrm{Z}$ and $k \mathrm{H}$ legs. All Z's except the first two are on-shell and longitudinally polarised; the Z's with momenta $q_{a, b}$ are off-shell and their Lorentz index is not resolved. The residue of the pole $\left(q_{a}+q_{j_{1}}+\cdots+q_{j_{m}}+h_{i_{1}}+\right.$ $\left.\cdots+h_{i_{\ell}}\right)^{-2}$ is given, up to a possible sign, by

$$
R=i K_{m+1, \ell}^{\alpha}\left(q_{a}\right) K_{n-1-m, k-\ell}^{\beta}\left(q_{b}\right)
$$

for even $m$, and by

$$
R=i K_{m, \ell+1}^{\alpha}\left(q_{a}\right) k_{n-2-m, k+1-\ell}^{\beta}\left(q_{b}\right)
$$

for odd $m$. Since $K_{n, k}^{\mu}(q)$ is proportional to $q^{\mu}$ except when $n=k=1$, each term in the $E^{0}$ terms of the amplitude is proportional to either $q_{a}^{\alpha}$ or $q_{b}^{\beta}$ or both. Upon contraction with polarisation vectors they therefore cancel. The single exception to this behaviour is $K_{1,1}\left(q_{a}\right)^{\alpha} K_{1,1}^{\beta}\left(q_{b}\right)$, that is the 4-point amplitude $\mathcal{M}(2 Z, 2 H)$ which stands unmasked as the most irregular amplitude of all in the $\mathrm{ZH}$ sector.

\section{On-shell recursion relations}

We have now proven the following: all $E^{2}$ contributions cancel in all $n$-point amplitudes with $n \geq 4$; terms with $E^{0} M^{2}$ only survive for $n=3$ or 4 ; and terms with $E^{0} m^{2}$ only occur in the 2Z,2H amplitude. For $n>4$ all amplitudes decrease with $E$ at least as fast as $E^{-1}$. To arrive at this conclusion we have used only that the external momenta are on-shell, and momentum conservation. To proceed further we consider on-shell decomposition relations, in the spirit of [18].

An $n$-point tree amplitude $\mathcal{M}$ contains $2^{n-1}-n-1$ internal propagators. Let $s$ be a set of $n_{s}$ of the external momenta (with $2 \leq n_{s} \leq n-2$ ), and let us call $p_{s}$ the total momentum of the set $s$. The corresponding propagator has denominator

$$
\Delta_{s}=p_{s}^{2}-m^{2}
$$

where $m$ is the $\mathbf{Z}$ or Higgs mass, as the case may be. We shall describe a deformation of the amplitude into a phase space of dimension 7 (a higher number is in principle also possible). 
The metric has signature $(+,-,-,-,+,-,-)$. The external momenta $k_{j}(j=1, \ldots, n)$ have of course no components in the extra dimension, nor does the auxiliary vector $t$ :

$$
\begin{aligned}
& k^{\mu}=\left(k^{0}, k^{1}, k^{2}, k^{3}\right) \quad \rightarrow \quad\left(k^{0}, k^{1}, k^{2}, k^{3}, 0,0,0\right), \\
& t^{\mu}=\left(t^{0}, t^{1}, t^{2}, t^{3}\right) \quad \rightarrow \quad\left(t^{0}, t^{1}, t^{2}, t^{3}, 0,0,0\right) .
\end{aligned}
$$

Now, we choose an arbitrary set of vectors $\eta_{j}(j=1, \ldots, n)$ having components only in the extra dimensions:

$$
\eta^{\mu}=\left(0,0,0,0, \eta^{5}, \eta^{6}, \eta^{7}\right)
$$

with the constraints

$$
\eta_{j}^{2}=0, \quad \sum_{j} \eta_{j}^{\mu}=0
$$

It is this requirement that necessitates the extra dimensions to number at least 3 ; but then we can always construct any number of such vectors. We define the following deformation, depending on a complex parameter $z$ :

$$
k_{j}{ }^{\mu} \rightarrow \tilde{k}_{j}^{\mu}=k_{j}{ }^{\mu}+z^{1 / 2} \eta_{j}{ }^{\mu}
$$

The longitudinal polarization vector of an external $\mathrm{Z}$ now automatically gets the deformation

$$
\epsilon_{j}{ }^{\mu} \rightarrow \tilde{\epsilon}_{j}^{\mu}=\epsilon_{j}{ }^{\mu}+\frac{z^{1 / 2}}{m} \eta_{j}{ }^{\mu}
$$

Note, however, that there are not 2 but 5 transverse polarisation vectors, with components in all dimensions. Just like the original $k_{j}$, the deformed $\tilde{k}_{j}$ are on-shell and the total momentum is conserved.

The deformed amplitude, $\mathcal{M}(z)$, is the original one, $\mathcal{M}(0)$, with the momenta and polarisations replaced by their deformed versions. It has denominators

$$
\tilde{\Delta}_{s}=\tilde{p}_{s}^{2}-m^{2}=\Delta_{s}+z \eta_{s}{ }^{2},
$$

which vanish at the $z$ value

$$
z_{s}=-\Delta_{s} / \eta_{s}{ }^{2}
$$

The residue at this pole is denoted $R\left(z_{s}\right)$. It is easily seen that $\mathcal{M}(z)$ is a rational function of $z$. Let us consider the limit $z \rightarrow \infty$. In this limit, the $\eta$ dominate the external momenta. Since the $E^{2}$ terms vanish, as do the $E^{1,0}$ terms for $n>4$,

$$
\mathcal{M}_{\infty} \equiv \lim _{z \rightarrow \infty} \mathcal{M}(z)=\left\{\begin{array}{cc}
\text { constant }, & n=4 \\
0, & n>4
\end{array}\right.
$$


We are therefore allowed the following contour integral manipulations:

$$
\begin{aligned}
\mathcal{M}(0) & =\frac{1}{2 \pi i} \oint_{z \sim 0} d z \frac{\mathcal{M}(z)}{z} \\
& =\mathcal{M}_{\infty}-\sum_{s} \frac{1}{2 \pi i} \oint_{z \sim z_{s}} d z \frac{R\left(z_{s}\right)}{z \tilde{\Delta}_{s}} \\
& =\mathcal{M}_{\infty}-\sum_{s} \frac{1}{2 \pi i} \oint_{z \sim z_{s}} d z \frac{R\left(z_{s}\right)}{z\left(z-z_{s}\right) \eta_{s}{ }^{2}} \\
& =\mathcal{M}_{\infty}+\sum_{s} \frac{R\left(z_{s}\right)}{\Delta_{s}} .
\end{aligned}
$$

Since at the pole $z=z_{s}$ the deformed momentum $\tilde{p}_{s}$ is exactly on-shell, and since for $\mathrm{Z}$ propagators in the unitary gauge

$$
\left\lfloor-g^{\mu \nu}+\frac{1}{m^{2}} \tilde{p}_{s}^{\mu} \tilde{p}_{s}^{\nu}\right\rfloor_{z=z_{s}}=\sum_{\lambda=1}^{6} \tilde{\epsilon}_{s}(\lambda)^{\mu} \tilde{\epsilon}_{s}(\lambda)^{\nu},
$$

where $\lambda$ denotes an enumeration of the (at least) six physical, normalised polarisation vectors, we see that $R\left(z_{s}\right)$ is precisely (a spin sum of) the product of two on-shell amplitudes:

$$
R\left(z_{s}\right)=A_{n_{s}+1} B_{n-n_{s}+1},
$$

where we have indicated the number of external legs in the factor amplitudes, which is always at most $n-1$. This allows us induction in $n$ : if both the on-shell amplitudes $A$ and $B$ respect unitarity in the sense that they have the correct behaviour with $E$, then

$$
\frac{R\left(z_{s}\right)}{\Delta_{s}} \sim \frac{1}{E^{2}} E^{4-\left(n_{s}+1\right)} E^{4-\left(n-n_{s}+1\right)}=E^{4-n} .
$$

Thus we have established that in the HZ model all on-shell tree amplitudes obey partialwave unitarity.

A final remark is in order here. We want to stress that in this paper we do not aim at computing the amplitudes, but rather want to study their high- $E$ behaviour. If we had opted for a two-line deformation, we would in the limit $|z| \rightarrow \infty$ have the situation of two high-energy particles moving in the background of lower-energy ones. The high$E$ limit would then be a situation like $m, M \ll q_{2, \ldots, n}, h_{2, \ldots, n} \ll z q_{1}, z h_{1}$, a problem in which two large ratios of scales occur. By using an all-line deformation we circumvent this artificial problem because the high- $E$ and high- $z$ limits actually conicide. Other all-line deformations have been used before [19-21], where the fact that Weyl spinors are used more or less enforces the restriction to four dimensions. Since we only consider vectors, the extension to higher dimensions is unproblematic. On the other hand, higher dimensions imply extra transverse polarisations, which are of course absent in a four-dimensional deformation. For internal lines, the only rôle of the extra polarisations is to ensure that the $\mathrm{Z}$ propagators remain in the unitary gauge; while for the external lines, only the 'original' three polarizations are present, albeit deformed. ${ }^{10}$

\footnotetext{
${ }^{10}$ We thank the referee for drawing our attention to this point.
} 


\section{Conclusions}

In this paper we prove the tree-level unitarity of all amplitudes in the Abelian Higgs model. This is not a new result: rather, it is the method of arriving at it that is of interest here, and we recapitulate the novelties involved.

- We have used only physical fields. The unitary gauge is widely considered inappropriate for studying unitarity (and renormalizability) because of its high-energy behaviour, but here we have shown that it actually forms the cornerstone of any treatment that aims at using physical degrees of freedom only: it provides the effective Feynman rules that led us to $Z_{n, k}$ and $H_{n, k}$.

- The Schwinger-Dyson equations of the theory are seen to lead to surprsingly simple forms for the off-shell amplitudes (cf. eq. (3.2)), which have to our knowledge not been obtained before.

- We have deformed the amplitudes by extending the dimensionality of phase space and deforming all lines simultaneously. We deem this all-line deformation necessary since we are dealing with a massive theory rather than unbroken YM-like theories in which the problem of relative scales does not enter.

Open Access. This article is distributed under the terms of the Creative Commons Attribution License (CC-BY 4.0), which permits any use, distribution and reproduction in any medium, provided the original author(s) and source are credited.

\section{References}

[1] P.W. Anderson, Plasmons, gauge invariance and mass, Phys. Rev. 130 (1963) 439 [InSPIRE].

[2] G. 't Hooft and M.J.G. Veltman, Regularization and renormalization of gauge fields, Nucl. Phys. B 44 (1972) 189 [INSPIRE].

[3] C. Becchi, A. Rouet and R. Stora, The Abelian Higgs-Kibble model. Unitarity of the $S$ operator, Phys. Lett. B 52 (1974) 344 [INSPIRE].

[4] H.G.J. Veltman, The equivalence theorem, Phys. Rev. D 41 (1990) 2294 [InSPIRE].

[5] C. Grosse-Knetter and R. Kögerler, Unitary gauge, Stuckelberg formalism and gauge invariant models for effective lagrangians, Phys. Rev. D 48 (1993) 2865 [hep-ph/9212268] [INSPIRE].

[6] H. Elvang and Y.-T. Huang, Scattering amplitudes, arXiv:1308.1697 [INSPIRE].

[7] S. Weinzierl, Tales of 1001 gluons, Phys. Rept. 676 (2017) 1 [arXiv: 1610. 05318] [InSPIRE].

[8] C. Itzykson and J.-B. Zuber, Quantum field theory, McGraw-Hill, New York U.S.A., (1980) [INSPIRE].

[9] M.E. Peskin and D.V. Schroeder, An introduction to quantum field theory, Addison-Wesley, Reading U.S.A., (1995) [INSPIRE].

[10] D. Bailin and A. Love, Introduction to gauge field theory, IOP and Adam Hilger, U.K., (1986) [INSPIRE]. 
[11] R. Kleiss, P4 lecture notes "paths, pictures, particles, processes", Radboud University, Nijmegen The Netherlands, (2017).

[12] J.M. Cornwall, D.N. Levin and G. Tiktopoulos, Uniqueness of spontaneously broken gauge theories, Phys. Rev. Lett. 30 (1973) 1268 [Erratum ibid. 31 (1973) 572] [INSPIRE].

[13] J.M. Cornwall, D.N. Levin and G. Tiktopoulos, Derivation of gauge invariance from high-energy unitarity bounds on the S matrix, Phys. Rev. D 10 (1974) 1145 [Erratum ibid. D 11 (1975) 972] [INSPIRE].

[14] E.N. Argyres, C.G. Papadopoulos and R.H.P. Kleiss, On amplitude zeros at threshold, Phys. Lett. B 302 (1993) 70 [hep-ph/9212280] [INSPIRE].

[15] E.N. Argyres, R.H.P. Kleiss and C.G. Papadopoulos, Nullification of multi-Higgs threshold amplitudes in the Standard Model, Phys. Lett. B 308 (1993) 315 [Addendum ibid. B 319 (1993) 544] [hep-ph/9303322] [INSPIRE].

[16] L.S. Brown, Summing tree graphs at threshold, Phys. Rev. D 46 (1992) R4125 [hep-ph/9209203] [INSPIRE].

[17] V.V. Khoze and M. Spannowsky, Higgsplosion: solving the hierarchy problem via rapid decays of heavy states into multiple Higgs bosons, Nucl. Phys. B 926 (2018) 95 [arXiv: 1704.03447] [INSPIRE].

[18] R. Britto, F. Cachazo, B. Feng and E. Witten, Direct proof of tree-level recursion relation in Yang-Mills theory, Phys. Rev. Lett. 94 (2005) 181602 [hep-th/0501052] [INSPIRE].

[19] N. Arkani-Hamed and J. Kaplan, On tree amplitudes in gauge theory and gravity, JHEP 04 (2008) 076 [arXiv:0801.2385] [INSPIRE].

[20] H. Elvang, D.Z. Freedman and M. Kiermaier, Proof of the MHV vertex expansion for all tree amplitudes in $N=4 S Y M$ theory, JHEP 06 (2009) 068 [arXiv:0811.3624] [INSPIRE].

[21] T. Cohen, H. Elvang and M. Kiermaier, On-shell constructibility of tree amplitudes in general field theories, JHEP 04 (2011) 053 [arXiv: 1010.0257] [INSPIRE]. 